\title{
Hemodynamic Evaluation of Vascular Reconstructive Surgery for Childhood Moyamoya Disease Using Single Photon Emission Computed Tomography
}

\author{
Shugo Takikawa, Hiroyasu Kamiyama, Hiroshi AbE, \\ Kenji MITSUMORI* and Mitsuo TSURU* \\ Department of Neurosurgery, Hokkaido University School of Medicine, Sapporo; \\ *Hokkaido Neurosurgical Memorial Hospital, Sapporo
}

\begin{abstract}
To evaluate the efficacy of vascular reconstructive surgery for childhood moyamoya disease, the cerebral blood flow (CBF) in 31 hemispheres of 16 patients was examined by single photon emission computed tomography (SPECT) using the ${ }^{133} \mathrm{Xe}$ inhalation method. Results were divided into two groups; 17 hemispheres with superficial temporal artery-middle cerebral artery (STA-MCA) anastomosis $[\mathrm{A}(+)$ group] and 14 hemispheres without anastomosis $[\mathrm{A}(-)$ group]. The mean hemispheric $\mathrm{CBF}(\mathrm{mCBF}$ ) and regional $\mathrm{CBF}(\mathrm{rCBF})$ in the frontal, temporal, occipital, and basal ganglia regions were calculated. Pre- and postoperative SPECT on the 10 hemispheres of the $\mathrm{A}(+)$ group showed an increase in $\mathrm{mCBF}$ in 6 hemispheres, the disappearance of the low perfusion area (LPA) in all 5 hemispheres where LPA was present before surgery, and an improvement in $\mathrm{rCBF}$ distribution (an increase in $\mathrm{rCBF}$ in the frontal and temporal lobes and a decrease in the basal ganglia). This suggests that vascular reconstruction is greatly effective in treating this disease. A comparison between the $\mathrm{A}(+)$ group and the $\mathrm{A}(-)$ group by postoperative SPECT, as well as the clinical outcomes and the postoperative findings of electroencephalography and angiography, revealed that the $\mathrm{A}(+)$ group was superior to the $\mathrm{A}(-)$ group in the frequency of L.PA $(12 \%$ and $43 \%$, respectively) and $\mathrm{rCBF}$ in the frontal region where STA-MCA anastomosis was usually performed. These results indicate that STA-MCA anastomosis with indirect synangiosis is the most effective treatment of childhood moyamoya disease.
\end{abstract}

Key words: moyamoya disease, childhood, vascular reconstructive surgery, STA-MCA anastomosis, cerebral blood How, single photon emission computed tomography

\section{Introduction}

The moyamoya disease of childhood is characterized by the progressive arterial stenosis or occlusion of the circle of Willis and by abnormal vascular collateral circulation networks at the base of the brain. The pathogenesis of this disease is presently unknown and basic treatment for it has yet to be established. Therefore, in order to prevent cerebral ischemia, vascular reconstructive surgery is performed to increase the blood supply to the ischemic brain from the external carotid system. To evaluate the efficacy of the vascular reconstructive surgery, the pre- and postoperative cerebral blood flow (CBF)

Received November 28, 1988; Accepted October 26,1989 was examined by single photon emission computed tomography (SPECT). The effectiveness of direct versus indirect vascular reconstruction was also investigated by the postoperative $\mathrm{CBF}$ study, the clinical outcomes, and electroencephalographic (EEG) and angiographic findings.

\section{Materials and Methods}

I. Pre- and postoperative hemodynamic changes CBF was measured on 10 hemispheres in 6 children with moyamoya disease (Cases 1-6) pre- and postoperatively (Table 1 ). They included three boys and three girls and their average age was 9.7 years (range, 7-13 years). All of them had experienced ischemic episodes, clinically diagnosed as transient ischemic attack (TIA) in five cases and a minor com- 
Table 1 Summary of 16 cases of moyamoya disease

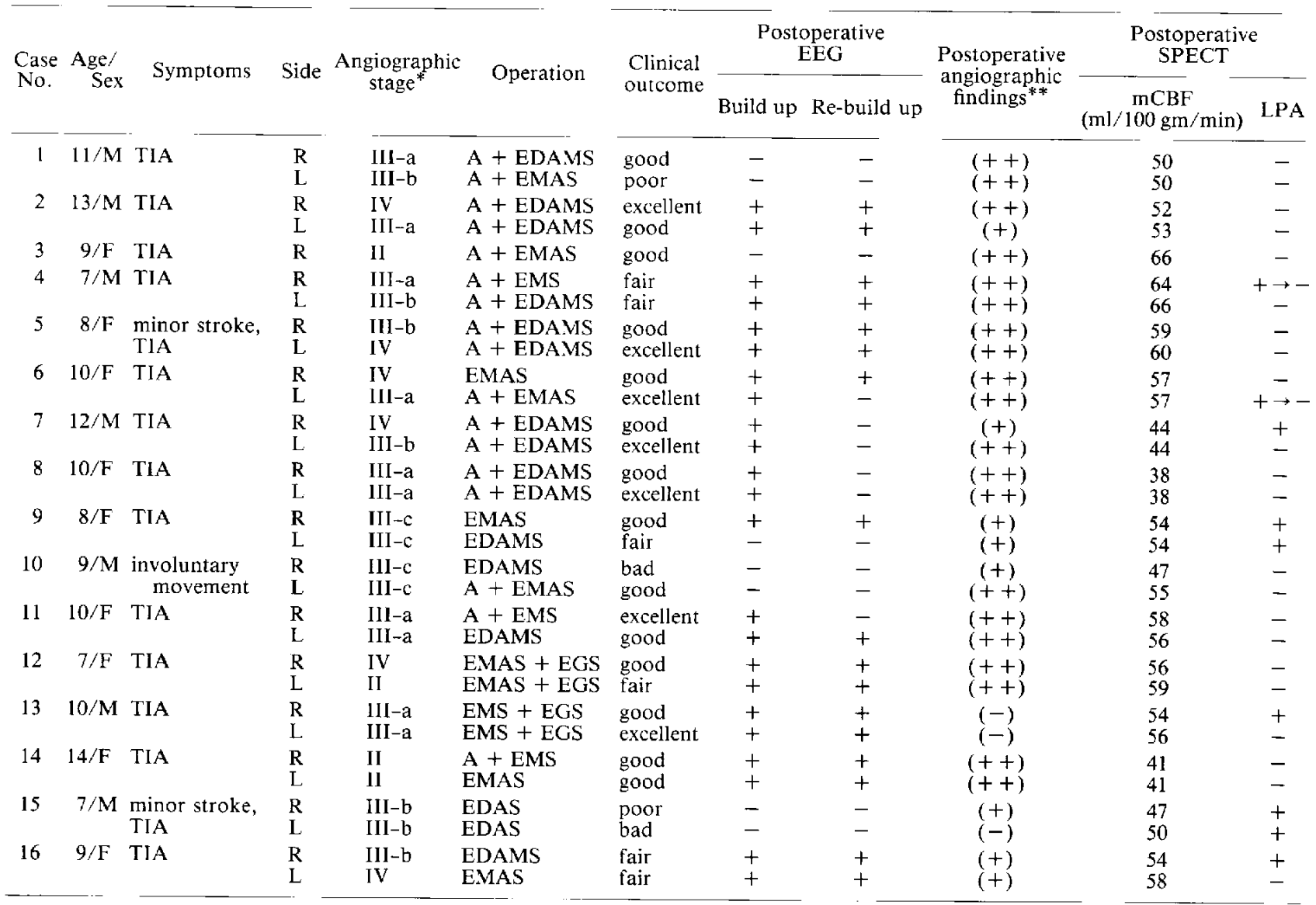

*According to Suzuki ${ }^{21)}$ and Fukuyama et al. ${ }^{5)}$ In Cases 1-6 SPECT was performed before and after operation, but in Cases 7-16 only postoperative SPECT was performed. EEG: electroencephalography, SPECT: single photon emission computed tomography, mCBF: mean hemispheric cerebral blood flow, LPA: low perfusion area, TIA: transient ischemic attack, A: superficial temporal artery (STA)-middle cerebral artery (MCA) anastomosis, EDAMS: encephaloduro-arterio-myo-synangiosis, EMAS: encephalo-myo-arterio-synangiosis, EDAS: encephalo-duro-arterio-synangiosis, EMS: encephalo-myo-synangiosis, EGS: encephalo-galeo-synangiosis, +: present, - : absent. **STA opacification: $(++)$, cortical branches of the MCA were visualized via the STA with enlargement of the STA diameter; $(+)$, cortical branches of MCA were visualized via the STA without enlargement of the STA diameter; $(-)$, no cortical branch of MCA was visualized via the STA.

pleted stroke in one. Computed tomographic (CT) scans revealed no abnormal low density areas in any case except one hemisphere. Angiographic stages ranged from stage II to stage IV as defined by the Suzuki and Fukuyama classification..$^{5,21)}$ All six patients underwent superficial temporal artery middle cerebral artery (STA-MCA) anastomosis with indirect vascular reconstruction such as encephaloduro-arterio-myo-synangiosis (EDAMS), encephalomyo-arterio-synangiosis (EMAS), and encephalomyo-synangiosis (EMS).

$\mathrm{CBF}$ was measured by the ${ }^{133} \mathrm{Xe}$ inhalation method with a circular detector array SPECT (Headtome SET-031, Shimadzu Co., Kyoto). The mean hemispheric $\mathrm{CBF}(\mathrm{mCBF})$ and regional $\mathrm{CBF}$ (rCBF) in the frontal, temporal, occipital, and basal ganglia regions were calculated at the slice $55 \mathrm{~mm}$ above and parallel to the orbito-meatal line, according to the "sequence of picture method" described by Kanno and Lassen. ${ }^{8)}$ The spatial resolution was $20 \mathrm{~mm}$ horizontally and $24 \mathrm{~mm}$ axially, measured as full width at half maximum. The area of low density on the CT scan was excluded from the region of interest on SPECT.

$\mathrm{CBF}$ was measured preoperatively at least 2 weeks after the ischemic attack, and postoperatively 2 or 3 times in each case, between day 13 and day 301 following the operation. 


\section{Comparison of operative effects}

Thirty-one hemispheres in 16 children with moyamoya disease with vascular reconstructive operations in our department since 1982 were analyzed. They contained seven boys and nine girls and ranged in age from 7 to 14 years (average, 9.6 years). The postoperative follow-up period ranged from 4 to 63 months (average, 26.4 months). The clinical details are listed in Table 1. They were divided into two groups: 17 hemispheres with STA-MCA anastomosis $[\mathrm{A}(+)$ group] and 14 hemispheres without STAMCA anastomosis [A(-) group]. The clinical outcomes and postoperative findings of EEG, angiography, and SPECT were compared between the two groups. Until 1985, indirect reconstruction had been performed only to minimize the surgical intervention except in cases with frequent TIAs requiring STA-MCA anastomosis. But recently STA-MCA anastomosis has also been carried out in addition to indirect reconstruction in all cases except those in which the STA supplies blood to the brain via transdural anastomosis. In addition, Preoperative angiographic stages significantly differ between the two groups (Table 1). Therefore, we consider it unlikely that the $\mathrm{A}(-)$ group contained a greater number of severe cases in which direct anastomosis was impossible.

The clinical outcomes were classified by frequency of ischemic attacks in postoperative period. Excellent was no TIA; good, a few TIAs immediately after operation; fair, TIAs less frequently than preoperatively; poor, TIAs as frequently as preoperatively; and bad, development of cerebral infarction.

EEG was recorded by monopolar leading with a $10 / 20$ electrode system and evaluated by the appearance of a "build up" phenomenon during hyperventilation and of a "re-build up" phenomenon after hyperventilation.

Conventional angiography was performed before and after surgery in all cases. The postoperative changes of STA opacification were evaluated on the lateral view of the external carotid angiograms and graded as follows: $(++)$ and $(+)$ denote the cortical branches of the MCA as visualized via the STA with or without considerable (more than double) enlargement of the STA diameter, respectively, and (-) indicates no cortical branch of the MCA as visualized via the STA.

CBF was measured by the same method as mentioned above. The $\mathrm{MCBF}$ value, the site of low perfusion area (LPA), and the relative $\mathrm{rCBF}$ distribution were compared between the two groups.

\section{Results}

\section{Postoperative changes in CBF}

Table 2 shows the mCBF values and the occurrence of LPA before and after the operation. The $\mathrm{mCBF}$ increased after surgery in 6 of the 10 hemispheres, and all the LPAs present in 5 of the 10 hemispheres before surgery disappeared after surgery. The transient enlargement of LPA, however, appeared early in the postoperative course in two hemispheres. As a result, the operation was effective in 9 of 10 hemispheres. The pre- and postoperative percentage ratios of $\mathrm{rCBF}$ to $\mathrm{mCBF}$ in different regions

Table 2 Pre- and postoperative changes of $\mathrm{mCBF}$ and LPA

\begin{tabular}{|c|c|c|c|c|c|c|}
\hline \multirow{2}{*}{$\begin{array}{l}\text { Case } \\
\text { No. }\end{array}$} & \multirow{2}{*}{ Side } & \multicolumn{2}{|c|}{$\begin{array}{c}\mathrm{mCBF} \\
(\mathrm{ml} / 100 \mathrm{gm} / \mathrm{min})\end{array}$} & \multicolumn{2}{|c|}{ LPA } & \multirow[t]{2}{*}{ Effect ${ }^{*}$} \\
\hline & & Preop & Postop & Preop & Postop & \\
\hline \multirow[t]{2}{*}{1} & $\mathbf{R}$ & 58 & 50 & + & - & + \\
\hline & $\mathrm{L}$ & 59 & 50 & + & - & + \\
\hline \multirow[t]{2}{*}{2} & $\mathrm{R}$ & 34 & 52 & + & - & ++ \\
\hline & $\mathrm{L}$ & 42 & 53 & - & - & + \\
\hline 3 & $\mathrm{R}$ & 57 & 66 & - & - & + \\
\hline \multirow[t]{2}{*}{4} & $\mathrm{R}$ & 58 & 64 & - & $+\rightarrow-$ & + \\
\hline & $\mathbf{L}$ & 58 & 66 & - & - & + \\
\hline \multirow[t]{2}{*}{5} & $\mathrm{R}$ & 57 & 59 & - & - & - \\
\hline & $\mathbf{L}$ & 51 & 60 & + & - & ++ \\
\hline 6 & $\mathbf{L}$ & 60 & 57 & + & $+\rightarrow-$ & + \\
\hline
\end{tabular}

*Effect: ++ , markedly improved; + , improved; - , no change.

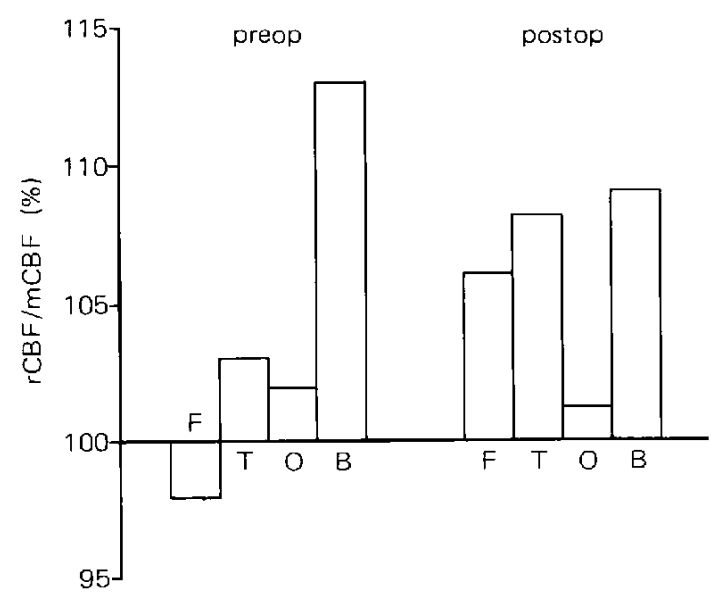

Fig. 1 Pre- and postoperative percentage ratios of $\mathrm{rCBF}$ to $\mathrm{mCBF}$ in different regions. $\mathrm{F}$ : frontal region, $\mathrm{T}$ : temporal region, $\mathrm{O}$ : occipital region, B: basal ganglia. 

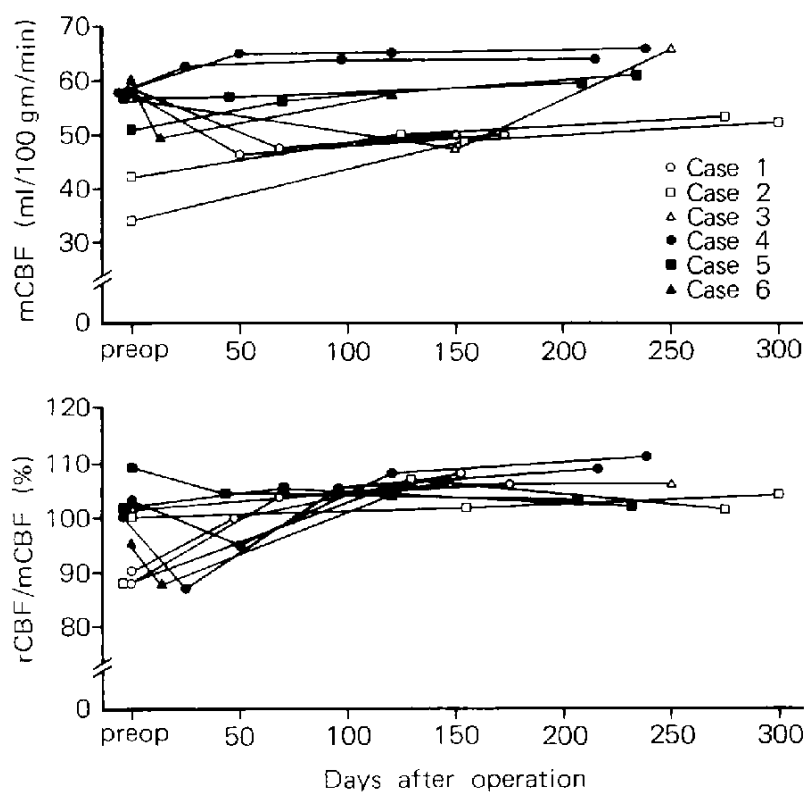

Fig. 2 Postoperative serial changes in mCBF (upper) and frontal rCBF ratio to $\mathrm{MCBF}$ (lower).

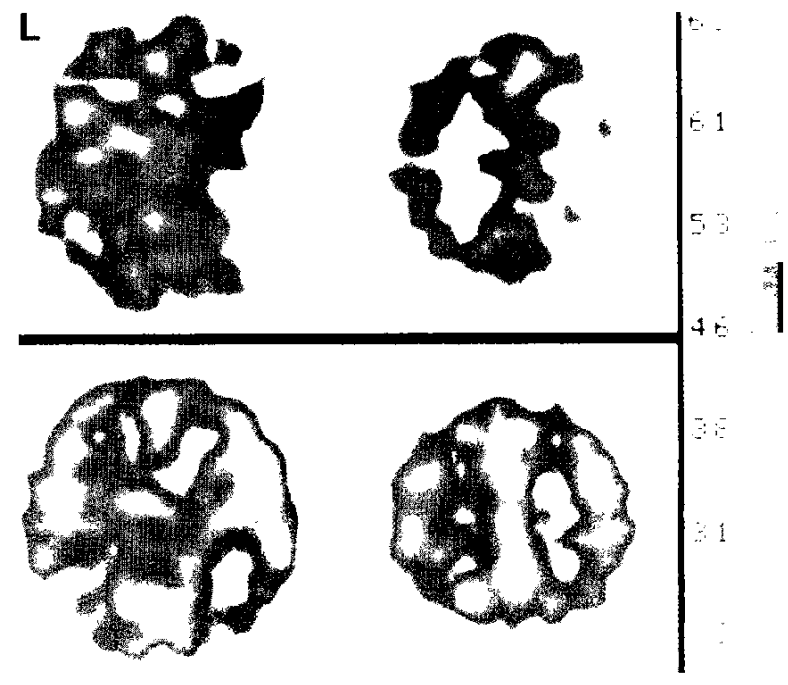

Fig. 3 Case 2. upper: Preoperative SPECT showing diffuse low perfusion area (I.PA) in the right middle cerebral artery territory. lower: Postoperative SPECT demonstrating no L.PA and bilateral increase of $\mathrm{mCBF}$.

are shown in Fig. 1, which shows that preoperatively the $\mathrm{rCBF}$ in the frontal, temporal, and occipital regions were almost the same as the $\mathrm{MCBF}$, while the $\mathrm{rCBF}$ at the basal ganglia was higher than the $\mathrm{mCBF}$. Postoperatively, the $\mathrm{rCBF}$ at the frontal and temporal regions close to the operative field in-
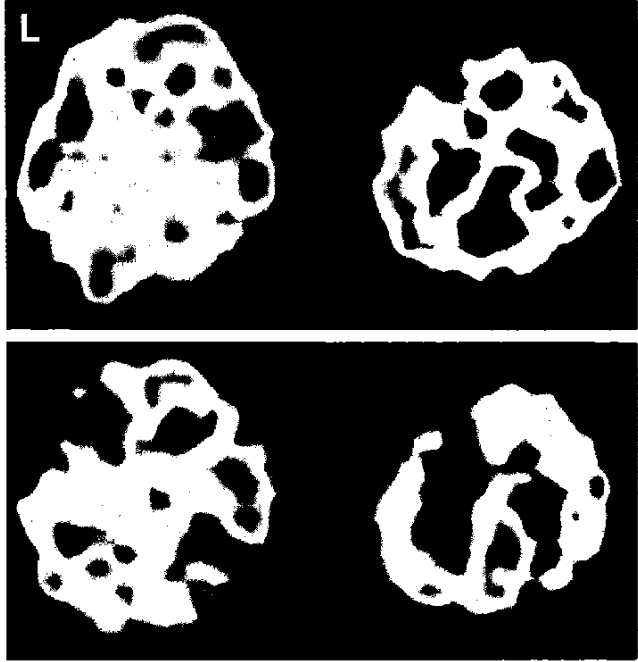

63

72
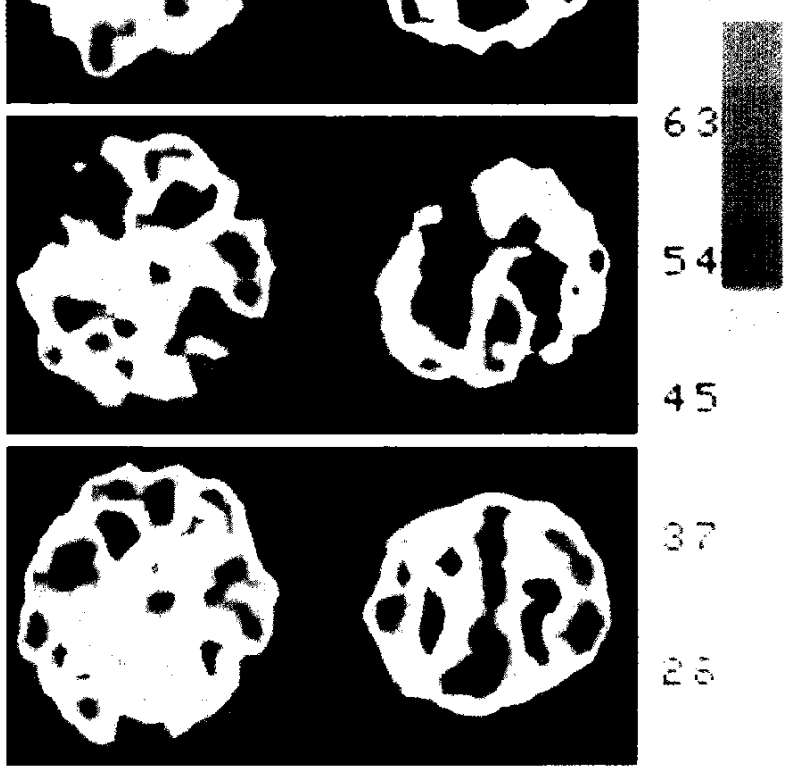

45

37

E5

Fig. 4 Case 6. upper: Preoperative SPECT showing local LPA in left frontal lobe. middle: Thirteen days after operation, bilateral reduction of $\mathrm{mCBF}$ and enlargement of the LPA are shown. lower: Recovery of $\mathrm{mCBF}$ and disappearance of LPA are revealed on 118 days after operation.

creased and the $\mathrm{rCBF}$ at the basal ganglia relatively decreased, which suggests an equilibration of postoperative rCBF distribution.

Some cases revealed a decrease of $\mathrm{mCBF}$ at an early stage after the operation, but afterwards it increased gradually, as shown in Fig. 2 upper. Such serial changes, a temporary deterioration followed by improvement, was more apparent in the relative $\mathrm{rCBF}$ at the frontal region which is closer to the operative field (Fig. 2 lower).

Two representative cases are described below.

Case 2 (Fig. 3): A 13-year-old boy suffered frequent TIAs on the left side from the age of 6 . Neurological examination and CT did not show any abnormalities. Preoperative SPECT showed a diffuse LPA in the right MCA territory and decreased $\mathrm{mCBF}$ on the right side. STA-MCA anastomosis with EDAMS was performed on both sides. No TIA occurred following the surgery and SPECT on postoperative day 128 showed the disappearance 
of the LPA and an increase of $\mathrm{mCBF}$ on both sides.

Case 6 (Fig. 4): A 10-year-old girl had a generalized convulsion at 8 years of age and underwent EMAS on the right side after the diagnosis of moyamoya disease. After the operation, she frequently suffered TIAs on the right side. A CT scan revealed no low-density area, but SPECT disclosed LPA in the left frontal lobe. A STA-MCA anastomosis with EMAS was performed on the left side. The postoperative course was uneventful without TIA. On postoperative day 13 SPECT showed an enlargement of the LPA and a reduction in mCBF on both sides. However, the LPA disappeared and the mCBF recovered by day 118 .

\section{Comparison of operative effects}

As summarized in Table 3 , of the clinical outcomes, $82 \%$ of cases in the $\mathrm{A}(+)$ group had an excellent or good postoperative course, while only $50 \%$ in the $\mathrm{A}(-)$ group. Moreover, in the $\mathrm{A}(-)$ group only, $14 \%$ ( 2 hemispheres) developed cerebral infarction even after the operation.

On postoperative EEG, the build up phenomenon was observed in about $70 \%$ of both groups, but the frequency of the re-build up phenomenon was definitely different between the two groups; $41 \%$ in the $\mathrm{A}(+)$ group and $71 \%$ in the $\mathrm{A}(-)$ group.

The postoperative angiography showed the cortical branches of the MCA were opacified via the STA in all of the $\mathrm{A}(+)$ group. Furthermore, most patients $(88 \%)$ showed considerable enlargement of the STA diameter compared with the preoperative angiography. On the other hand, only $36 \%$ in the

Table 3 Postoperative findings

\begin{tabular}{|c|c|c|}
\hline & $\begin{array}{l}\text { astomosis }(+) \\
(\mathrm{n}=17)\end{array}$ & $\begin{array}{c}\text { Anastomosis }(-) \\
(\mathrm{n}=14)\end{array}$ \\
\hline \multicolumn{3}{|l|}{ Clinical outcome } \\
\hline excellent & $35 \%$ & $7 \%$ \\
\hline good & 47 & 43 \\
\hline fair & 12 & 29 \\
\hline poor & 6 & 7 \\
\hline bad & 0 & 14 \\
\hline \multicolumn{3}{|l|}{ EEG findings } \\
\hline build up (+) & $76 \%$ & $71 \%$ \\
\hline re-build up (+) & 41 & 71 \\
\hline \multicolumn{3}{|l|}{ Angiographic findings } \\
\hline *STA opacification $(t+)$ & $88 \%$ & $36 \%$ \\
\hline$(+)$ & 12 & 43 \\
\hline$(-)$ & 0 & 21 \\
\hline \multicolumn{3}{|l|}{ SPECT findings } \\
\hline $\mathrm{mCBF}(\mathrm{ml} / 100 \mathrm{gm} / \mathrm{min})$ & $53 \pm 9$ & $53 \pm 5$ \\
\hline $\operatorname{LPA}(+)$ & $12 \%$ & $43 \%$ \\
\hline
\end{tabular}

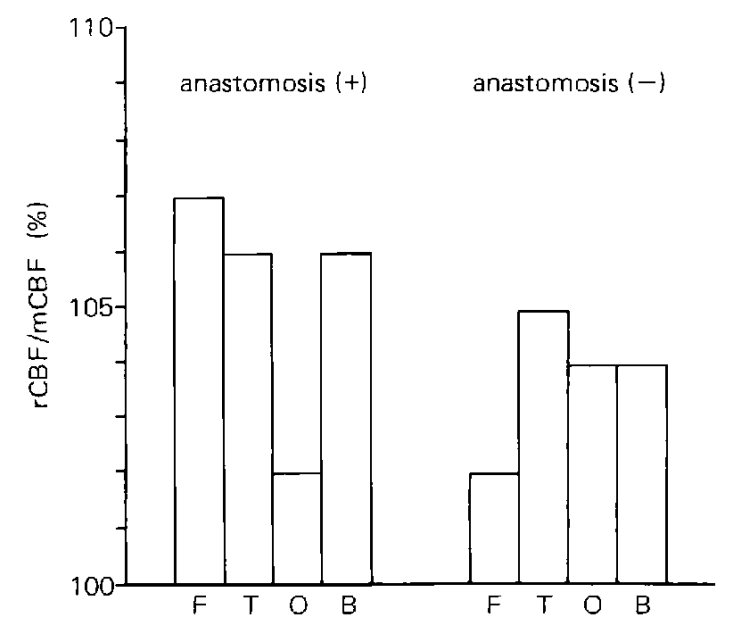

Fig. 5 Postoperative percentage ratios of $\mathrm{rCBF}$ to $\mathrm{mCBF}$ in different regions.

$A(-)$ group showed considerable enlargement of STA, and no cortical branch was visualized via the STA in three cases $(21 \%)$.

Postoperative SPECT revealed no difference in the $\mathrm{mCBF}$ between the two groups. LPAs were found in only $12 \%$ in the $\mathrm{A}(+)$ group, in contrast to $43 \%$ in the $\mathrm{A}(-)$ group. Moreover, in the $\mathrm{A}(+)$ group, LPAs were not present in the MCA territory, only in the posterior cerebral artery territory. Most LPAs in the $A(-)$ group included the MCA territory. The relative $\mathrm{rCBF}$ distribution is shown in Fig. 5. The rCBF at the frontal region in the $\mathrm{A}(+)$ group was higher than that in the $\mathrm{A}(-)$ group.

\section{Discussion}

The treatment of moyamoya disease remains controversial. However, surgical treatment such as vascular reconstruction has gradually gained wide acceptance since it was reported by Krayenbühl, ${ }^{13)}$ Karasawa et al., ${ }^{9,10)}$ and Matsushima et al. ${ }^{15)}$ In the present study the efficacy of vascular reconstruction for childhood moyamoya disease is evaluated by cerebral hemodynamics using SPECT, which enables us to derive three-dimensional measurements of CBF.

Hemodynamic changes after vascular reconstruction in children with moyamoya disease was investigated by Karasawa et $a l .,{ }^{121}$ using the argon desaturation method. They reported a significant increase in the whole brain blood flow in patients with STA-MCA anastomosis or EMS. Using two-dimensional measurement by ${ }^{133} \mathrm{Xe}$ inhalation, Takeuchi $\mathrm{i}^{22)}$ observed an increase in mCBF in $14(67 \%)$ of 21 
hemispheres after EMAS. Similarly, Ishii et $a .^{7}$ observed increased $\mathrm{mCBF}$ in $21(54 \%)$ of 39 hemispheres. Using positron emission tomography, Fukui et al. ${ }^{4)}$ noted an improvement of rCBF in 12 $(63 \%)$ of 19 hemispheres mainly after EDAS. In our study, mCBF increased in $6(60 \%)$ of 10 hemispheres, and LPA disappeared in all 5 hemispheres in which LPA was present before surgery. In 9 out of 10 hemispheres an increase in mCBF or disappearance of LPA was observed, which suggests vascular reconstruction is effective in accordance with previous reports.

Gotoh et $a l .{ }^{6}$ reported the disappearance of "hyperfrontality" in moyamoya disease. In our case, preoperatively, "the hyperfrontality" was not found but rCBF at the basal ganglia, which is abundant in moyamoya vessels, was well preserved. Postoperatively rCBF increased in the frontal and temporal regions close to the operative field and decreased relatively at the basal ganglia. This corresponds to the postoperative angiographic changes where the collateral vascular channels via the external carotid system were visualized in the operative field and the moyamoya vessels diminished gradually. As a result, an equilibrating of $\mathrm{rCBF}$ distribution was observed as reported by Nishimoto et al. ${ }^{20}$ However, their findings contradict ours somewhat, in that $\mathrm{rCBF}$ increased mainly in the temporal region, and not in the frontal region. This discrepancy is thought to be a result of differences in operative procedure. Nishimoto et al. performed only EDAS or EDAMS with temporal craniotomy, while we made the craniotomy as extensive as possible and performed STA-MCA anastomosis in the frontal lobe.

Postoperative serial changes in $\mathrm{mCBF}$ and $\mathrm{rCBF}$ showed transient reduction soon after the operation as noted by previous reports. ${ }^{13,23)}$ The causes of this temporary deterioration seem to be the influence of general anesthesia on surgery and operative intervention such as the craniotomy itself, draining of cerebrospinal fluid, and temporary occlusion of the recipient artery during anastomosis.

As mentioned above, vascular reconstructive surgery is widely accepted for the treatment of moyamoya disease and several kinds of reconstruction are performed. A controversy remains as to which surgical procedure is the most effective. . $^{1-4,12,16,18,19}$, The operative approaches are divided into two groups; direct reconstruction such as STA-MCA anastomosis ${ }^{9,13)}$ and indirect reconstruction such as EMS, ${ }^{10)}$ EDAS, ${ }^{14)}$ and EMAS. Indirect reconstruction has the advantage that the surgical process is very simple and surgical intervention is minimal. Fukui et $a l{ }^{3)}$ reported, however, that it failed to provide a sufficient collateral blood supply in $20-30 \%$ of cases, in which ischemic symptoms persisted or progressed even after the operation. In such cases, Nagata et $a l .{ }^{18)}$ further performed direct anastomosis resulting in an improvement of the symptoms in all patients. They therefore concluded that direct anastomosis should be considered the best choice for the treatment. Moreover, it is said that indirect reconstruction cannot establish immediate revascularization and is not suitable for the patients with frequent TIAs. Abe and Kamiyama ${ }^{1)}$ investigated the preand postoperative clinical symptoms, EEG, and angiographic findings and found that STA was the most powerful source in the external carotid system supplying collateral blood flow, and that STA-MCA anastomosis, with an indirect nonanastomotic bypass, was more effective than only an indirect bypass. However, Karasawa et al., ${ }^{12)}$ using the argon desaturation method, observed an increase in $\mathrm{CBF}$ and cerebral oxygen metabolism 6 months after surgery in both the STA-MCA anastomosis group and the EMS group without any significant difference. In our study, the $\mathrm{A}(+)$ group was superior to the $\mathrm{A}(-)$ group in hemodynamics, as well as clinical outcomes and EEG and angiographic findings. In detail, the frequency of LPAs was higher in the $\mathrm{A}(-)$ group and most of the LPAs included the MCA territory where the operative effect is most expected in this sort of operation. This suggests insufficient collateral blood supply in the $\mathrm{A}(-)$ group. By contrast, the collateral flow seemed sufficient to eliminate the LPAs, at least in the MCA territory, in the $\mathrm{A}(+)$ group. Furthermore, the rCBF in the frontal region in the $A(+)$ group was higher than that in the $\mathrm{A}(-)$ group, which also indicates the good effect of STA-MCA anastomosis in the frontal lobe.

We conclude that STA-MCA anastomosis with indirect vascular reconstruction is the most effective treatment for childhood moyamoya disease in spite of its disadvantages of technical difficulty and greater surgical intervention. However, it is impossible even for this surgical procedure to prevent ischemia in the posterior cerebral artery or anterior cerebral artery territory in some cases, and this requires further studies on the need for a more extensive vascular reconstruction such as omentum transplantation. ${ }^{11,14,17)}$

\section{References}

1) Abe H, Kamiyama H: Surgical treatment of the occlusion of the circle of Willis, in: Annual Report 1986 of the Research Committee on Spontaneous Occlusion of the Circle of Willis of the Ministry of Health and 
Welfare Japan. 1987, pp 37-42 (in Japanese)

2) Fujita K, Tamaki N, Matsumoto S: Surgical treatment of moyamoya disease in children: Which is more effective procedure, EDAS or EMAS? Childs Nerv Syst 2: 134-138, 1986

3) Fukui M, Matsushima $T$, Fujii $K$, Hasuo $K$, Kuwabara Y: Surgical treatment in pediatric cases of moyamoya disease, in: Annual Report 1987 of the Research Committee on Spontaneous Occlusion of the Circle of Willis of the Ministry of Health and Welfare Japan. 1988, pp 90-94 (in Japanese)

4) Fukui M, Matsushima T, Kuwabara W, Ichiya Y, Wada M, Hasuo K: Hemodynamic evaluation of moyamoya disease in children by positron emission computed tomography before and after neurosurgical procedures (EDAS, EMS, EMAS), in: Annual Report 1986 of the Research Committee on Spontaneous Occlusion of the Circle of Willis of the Ministry of Health and Welfare Japan. 1987, pp 9198 (in Japanese)

5) Fukuyama Y, Umezu R, Mitsuishi Y, Ono Y, Kakigi $\mathrm{Y}$ : Annual Report 1984 of the Research Committee on Spontaneous Occlusion of the Circle of Willis of the Ministry of Health and Welfare Japan. 1985, pp 28-33 (in Japanese)

6) Gotoh F, Fukuuchi Y, Takashima S, Kawamura J, Terayama Y: Local cerebral blood flow and $\mathrm{CO}_{2}$ responsiveness in patients with "moyamoya disease," in: Annual Report 1986 of the Research Commitiee on Spontaneous Occlusion of the Circle of Willis of the Ministry of Health and Welfare Japan. 1987, pp 78-87 (in Japanese)

7) Ishii $\mathrm{R}$, Takeuchi $\mathrm{S}$, Ibayashi $\mathrm{K}$, Tanaka R: Intelligence in children with moya-moya disease: Evaluation after surgical treatment to change in cerebral blood flow. Stroke 15: 873-877, 1984

8) Kanno I, Lassen NA: Two methods for calculating regional cerebral blood flow from emission computed tomography of inert gas concentrations. J Comput Assist Tomogr 3: 71-76, 1979

9) Karasawa J, Kikuchi H, Furuse S, Kawamura J, Sasaki T: Treatment of moyamoya disease with STAMCA anastomosis. $J$ Neurosurg 49: 679-688, 1978

10) Karasawa J, Kikuchi H, Furuse S, Sakaki T, Yoshida Y, Ohnishi H, Taki W: A surgical treatment of "moyamoya" disease "encephalo-myo synangiosis." Neurol Med Chir (Tokyo) 17 [Part I]: 29-37, 1977

11) Karasawa J, Kikuchi H, Kawamura J, Sakaki T: Intracranial transplantation of the omentum for cerebrovascular moyamoya disease. A two-year follow-up study. Surg Neurol 14: 444-449, 1980

12) Karasawa J, Kikuchi H, Kuriyama $Y$, Sawada $T$, Kuro M, Kobayashi K, Koike T, Mitsugi T: Cerebral hemodynamics in "moyamoya"' disease. II. Measurements of cerebral circulation and metabolism by use of the argon desaturation method in pre- and postneurosurgical procedures. Neurol Med Chir (Tokyo) 21: 1161-1168, 1981 (in Japanese)

13) Krayenbühl HA: The moyamoya syndrome and neurosurgeon. Surg Neurol 4: 353-360, 1975

14) Kikuchi H, Nagata I, Miyamoto S, Karasawa J: Omentum transplantation for the treatment of moyamoya disease, in: Annual Report 1986 of the Research Committee on Spontaneous Occlusion of the Circle of Willis of the Ministry of Health and Welfare Japan. 1987, pp 32-36 (in Japanese)

15) Matsushima Y, Fukai N, Tanaka K, Tsuruoka K, Inaba $\mathrm{Y}$, Aoyagi $\mathrm{M}$, Ohno $\mathrm{K}$ : A new surgical treatment of moyamoya disease in children. A preliminary report. Surg Neurol 15: 313-320, 1981

16) Matsushima $Y$, Yamaguchi $T$, Takasato $Y$, Tomida S, Fukumoto T, Suzuki R, Tomita H, Inaba $Y$ : Changes in symptoms after encephalo-duro-arteriosynangiosis (EDAS) in pediatric moyamoya disease. No To Hattatsu 18: 3-7, 1986 (in Japanese)

17) Miyamoto S, Kikuchi H, Karasawa J, Nagata I, Ihara I, Yamagata S: Study of the posterior circulation in moyamoya disease. Part 2: Visual disturbances and surgical treatment. J Neurosurg 65: 454-460, 1986

18) Nagata I, Kikuchi $H$, Miyamoto $S$ : Additional reconstructive surgery in the patients of moyamoya disease pretreated with unsuccessful encephalo-duroarterio-synangiosis (EDAS), in: Annual Report 1987 of the Research Committee on Spontaneous Occlusion of the Circle of Willis of the Ministry of Health and Welfare Japan. 1988, pp 95-98 (in Japanese)

19) Nakagawa $Y$, Gotoh S, Shimoyama M, Ohtsuka K, Mabuchi S, Sawamura $Y$, Abe $H$, Tsuru M: Reconstructive operation for moyamoya disease. Surgical indication for the hemorrhagic type, and preferable operative methods. Neurol Med Chir (Tokyo) 23: 464-470, 1983 (in Japanese)

20) Nishimoto A, Kuyama $H$, Kinugasa $K$, Niimi $H$, Murota T: Hemodynamic changes after vascular reconstructive surgery for moyamoya disease, in: Annual Report 1986 of the Research Committee on Spontaneous Occlusion of the Circle of Willis of the Ministry of Health and Welfare Japan. 1987, pp 99-104 (in Japanese)

21) Suzuki J, Takaku A: Cerebral vascular "Moyamoya" disease. A disease showing abnormal net-like vessels in base of brain. Arch Neurol 20: 288-299, 1969

22) Takeuchi S: Cerebral hemodynamics in patients with moyamoya disease (part II). A study of regional cerebral blood flow by ${ }^{133} \mathrm{Xe}$ inhalation method. Neurol Med Chir (Tokyo) 23: 720-728, 1983 (in Japanese)

23) Yonekawa Y, Okuno T, Handa H: "Moyamoya" disease: Clinical review and surgical treatment, in Fein JM, Flamm ES (eds): Cerebrovascular Surgery, vol 2. New York, Springer, 1985, pp 557-580

Address reprint requests to: $\mathrm{S}$. Takikawa, M.D., Department of Neurosurgery, Hokkaido University School of Medicine, North-15, West-7, Kita-ku, Sapporo 060, Japan. 